\title{
Excited muonium state in CdS
}

\author{
D. G. Eshchenko, ${ }^{1, *}$ V. G. Storchak, ${ }^{2}$ S. P. Cottrell, ${ }^{3}$ and S. F. J. Cox ${ }^{3}$ \\ ${ }^{1}$ Institute for Nuclear Research, Moscow 117312, Russia \\ ${ }^{2}$ Russian Research Centre “Kurchatov Institute,” Kurchatov Square 46, Moscow 123182, Russia \\ ${ }^{3}$ ISIS Facility, Rutherford Appleton Laboratory, Oxfordshire OX11 OQX, United Kingdom \\ (Received 13 February 2003; revised manuscript received 13 June 2003; published 18 August 2003)
}

\begin{abstract}
The process of electron capture by a positive muon in semi-insulating CdS has been studied using $\mu \mathrm{SR}$ techniques in electric fields. Weak electric fields of about $8 \mathrm{kV} / \mathrm{cm}$ are sufficient to suppress the muonium formation, suggesting electron capture into excited hydrogenic states. The data indicate the first excited $(n$ =2) state to be the bottleneck to subsequent relaxation to the ground $(n=1)$ state, serving as a guide to electron capture by an interstitial proton into shallow-donor state of the hydrogen impurity in a wide-gap semiconductor.
\end{abstract}

DOI: 10.1103/PhysRevB.68.073201

PACS number(s): 72.20.Jv, 76.75. $+\mathrm{i}$

Since the discovery in the early 1980 's that atomic hydrogen can bind shallow impurities in semiconductors and thus passivate their electrical and optical activities the structure and dynamics of hydrogen isotopes in semiconductors have been the subject of intense studies (for a recent review see Ref. 1). However, while considerable information was accumulated on hydrogenic-impurity complexes, relatively little is known about isolated hydrogen states in semiconductors due to high mobility and reactivity of $\mathrm{H}$ atom down to very low temperature. For observation of the neutral paramagnetic centres, muonium studies are at a particular advantage. Muonium $\left(\mathrm{Mu}=\mu^{+} e^{-}\right)$is commonly formed when positive muons are implanted at low temperatures in semiconductors. The muonium atom has a reduced mass almost the same as that of $\mathrm{H}$, its electronic states and chemical interactions are literally those of a light hydrogen isotope $\left(m_{\mu} \simeq m_{p} / 9\right)$. It may be detected and characterized with great sensitivity via $\mu \mathrm{SR}$ (muon spin-rotation) spectroscopy, ${ }^{2}$ the short muon lifetime $\left(\tau_{\mu}=2.2 \times 10^{-6} \mathrm{~s}\right)$ setting a time scale which favors population of intrinsic interstitial sites, before diffusion occurs to other defects or impurities. It was thus accepted ${ }^{3}$ that the study of muonium in semiconductors deals with "early history" of a hydrogenlike impurity. For a recent review on the muonium behavior in IV-IV and III-V compounds see Ref. 4.

The situation now is that new types of wide-gap semiconductors, including the II-VI compounds, are on the verge of important applications. Defects and impurities are found to play particularly important roles in these new materials. While hydrogen is by no means the only such impurity, it is certainly one of the most significant, controlling the mechanism of deposition and doping and affecting final electronic properties. Until now little is know about hydrogen impurities in II-VI semiconductors. In the II-VI compound $\mathrm{ZnO}$, some early electrical measurements were taken as evidence that hydrogen could, in this wider-gap material, act as a dopant in its own right. ${ }^{5,6}$ These behavior was confirmed recently by an observation of muonium centers in $\mathrm{CdS}, \mathrm{CdSe}$, CdTe, and $\mathrm{ZnO}$ compounds. ${ }^{7}$

Of relevance to the present work is the fact that electrons (and holes) created during the process of muon implantation and thermalization are available for interaction and capture, irrelevant of temperature or doping. Muon spin relaxation experiments with electric fields ${ }^{8-11}$ have shown that in condensed samples the stopped muon is very close to (at distances less than $10^{-5} \mathrm{~cm}$ ) or even surrounded by its own track products. ${ }^{12,13}$ Some of the excess electrons generated in the track are close enough to reach and to be captured by the stopped muon and form a muonium atom. This process of "delayed" muonium formation (DMF) is relevant to the process of trapping of excess electrons by an attractive center. In this report we present evidence that the positive muon $\left(\mu^{+}\right)$ may be treated as a trapping center in some semiconductors.

In order to understand the process of an electron capture by a positive center, one must remember that the electron not only must come to the vicinity of the center, it must on arrival perform the unlikely task of depositing perhaps many times the Debye energy (and it takes several phonons to carry away this energy). The cascade model of the electron capture $^{14,15}$ avoids this problem. The key point is as follows: whereas multiphonon transitions to the ground state of the trap yield cross sections five or ten orders of magnitude too small, ${ }^{16}$ capture into excited states with a large radius followed by a cascade of one-phonon transitions leads to cross sections of the right order of magnitude. ${ }^{15}$ The initial capture involves one phonon and takes place into one of very excited orbits. Subsequent collisions may eject the electron or cause it to increase its binding energy. The "sticking probability" or probability of eventual capture into the ground state becomes significant for binding energies of order kT. As the temperature is reduced, capture into orbits of larger radius becomes effective and the cross section therefore increases rapidly with decreasing temperature.

From this scenario it is clear that the temperature dependence of the muonium fraction measured in $\mu \mathrm{SR}$ experiments (this fraction could be treated as the "sticking probability" or probability of eventual capture of a free electron into the ground muonium state) does not necessarily deal with thermal ionization from the ground state but may include thermal ionizations from excited muonium states. In this case, both the ionization temperature and the characteristic electric field will be significantly reduced from those 
required to ionize deep states. In particular, the characteristic electric field is expected to be much less than electric fields of the atomic scale $\left(\sim 10^{9} \mathrm{~V} / \mathrm{cm}\right)$.

To understand the process of the electron capture by a positive attractive center in a semiconductor $\mu$ SR experiments using external electric fields have proved very useful: ${ }^{13,17,18}$ With increasing electric field those excited states for which the bias across the orbit is bigger than the binding energy ${ }^{19}$

$$
2 E e a_{n}>U_{n}
$$

(where $E$ is the external electric field, $e$ is the electron charge, $a_{n}$ is the radius, and $U_{n}$ is the binding energy of the $n$th excited state) are removed from the excitation spectrum and the number of "valid" excited states is decreased. Therefore, by scanning the electric field at any given temperature one can find the "bottleneck" or the excited state responsible for the electron capture to the ground muonium state.

We used the technique of $\mu$ SR experiments in external electric fields to prove that the electron capture by the muon in CdS semiconductor and eventual formation of the ground muonium state proceeds through an excited muonium state (or states). Time-differential $\mu$ SR experiments were performed on the EMU beam line at the ISIS Pulsed Muon Facility at the Rutherford Appleton Laboratory. A $15 \mathrm{~mm}$ $\times 15 \mathrm{~mm} \times 1 \mathrm{~mm}$ polycrystalline intrinsic $\mathrm{CdS}$ sample was studied, both surfaces were covered with vapor-deposited silver electrodes to a thickness of a few nm. Experimental data were obtained using the technique of $\mu \mathrm{SR}$ measurements in frequently switched external electric fields. ${ }^{17}$ This method is used to avoid the influence of possible accumulation of a space charge near the sample surface. Such a charge build-up can screen the external electric field and reduce (if not cancel) the effect under study. The shorter the switching time the less charge accumulates, thereby improving the accuracy of the experiment. For the particular CdS sample and muon beam conditions used at ISIS, the switching period should not exceed $10 \mathrm{~s}$.

The remarkable feature of the muonium state observed in CdS (Ref. 20) is that the hyperfine interaction $A$ is extremely small amounting only about $10^{-4}$ of the vacuum value. For this state even a modest magnetic field of $0.01 \mathrm{~T}$ (the field used for this experiment) sets up the high field limiting case with muonium frequencies positioned symmetrically about the diamagnetic signal. ${ }^{2}$ Therefore, the experimental muon decay asymmetry ${ }^{2}$ was fitted to the sum of one diamagnetic and two muonium signals

$$
\begin{aligned}
A_{0} P(t)= & A_{D} \exp \left(-\lambda_{D} t\right) \cos \left(\omega_{\mu} t+\varphi_{D}\right) \\
& +\frac{1}{2} A_{\mathrm{Mu}} \exp \left(-\sigma_{\mathrm{Mu}}^{2} t^{2}\right) \cos \left(\omega_{12} t+\varphi_{\mathrm{Mu}}\right) \\
& +\frac{1}{2} A_{\mathrm{Mu}} \exp \left(-\sigma_{\mathrm{Mu}}^{2} t^{2}\right) \cos \left(\omega_{34} t+\varphi_{\mathrm{Mu}}\right),
\end{aligned}
$$

where $A_{0}$ is the normalization factor (the maximum muon decay asymmetry), $A_{D}$ and $A_{\mathrm{Mu}}, \lambda_{D}$, and $\sigma_{\mathrm{Mu}}, \omega_{\mu}$, and $\omega_{12}$ and $\omega_{34}, \varphi_{D}$, and $\varphi_{\mathrm{Mu}}$ are the diamagnetic and muo-

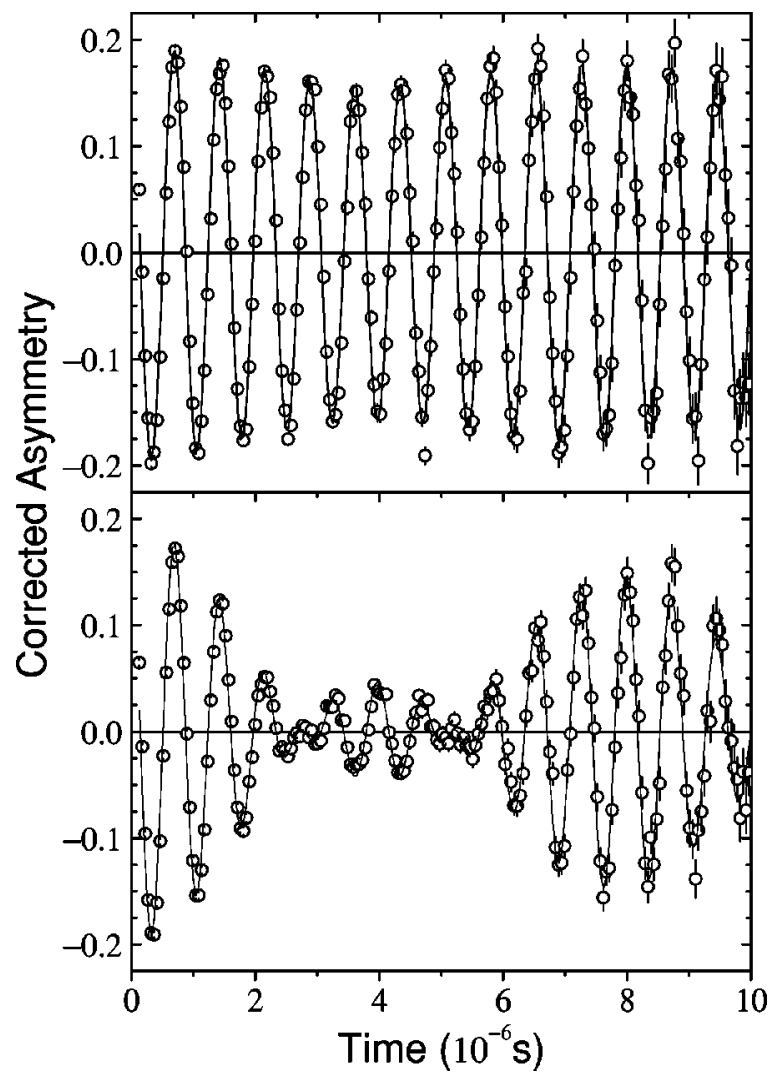

FIG. 1. Experimental corrected asymmetry $\mu$ SR spectra in intrinsic polycrystalline CdS sample at $T=11 \mathrm{~K}$ in transverse magnetic field $B=0.01 \mathrm{~T}$. Top: high external electric field $20 \mathrm{kV} / \mathrm{cm}$. Big diamagnetic plus very small muonium signal is seen. Bottom: zero electric field. Theoretical curve is the sum of two muonium frequencies plus a small diamagnetic signal.

nium asymmetries, relaxation rates, frequencies and initial phases, respectively. Muonium and diamagnetic signals in $\mathrm{CdS}$ are temperature dependent. ${ }^{20}$ At low temperatures the muonium signal dominates, muonium fraction decreases at the expense of the diamagnetic fraction as the temperature is increased, although the temperature dependence is weak up to $15 \mathrm{~K}$. To reduce the influence of thermal processes the electric field experiments were performed at $11 \mathrm{~K}$.

Both muonium and diamagnetic signals in $\mathrm{CdS}$ are strongly influenced by the external electric field. Figure 1 presents experimental spectra in the CdS sample at $T$ $=11 \mathrm{~K}$ in a transverse magnetic field $B=0.01 \mathrm{~T}$ for zero electric field (bottom) and electric field $E=20 \mathrm{kV} / \mathrm{cm}$ (top). One can see a multifrequency signal (a superposition of the diamagnetic and two muonium frequencies; muonium signal dominates) for $E=0$; this beating is almost absent at high electric fields where the diamagnetic asymmetry approaches to 0.2 - close to the maximum muon decay asymmetry.

Electric field dependences of both the diamagnetic and muonium signals in CdS sample are shown in Fig. 2. The direction of the electric field coincides with the direction of the initial muon beam momentum, the half-period of the electric field switching is $5 \mathrm{~s}$, the range of the electric field we applied is up to $20 \mathrm{kV} / \mathrm{cm}$. The mere fact that the sample 


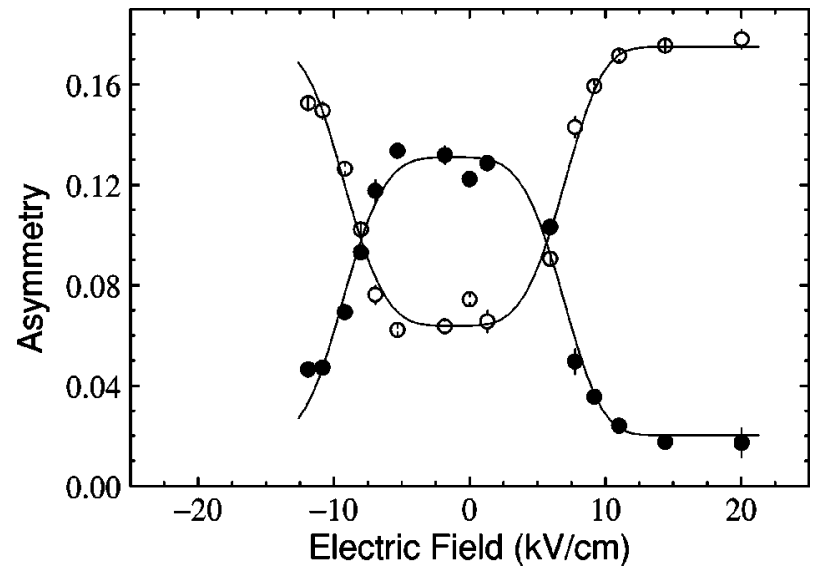

FIG. 2. Electric field dependence of the diamagnetic asymmetry (open circles) and muonium asymmetry (filled circles) in intrinsic polycrystalline CdS at $T=11 \mathrm{~K}$. Positive electric field is in the direction of the initial muon beam momentum. The half-period of the alternating electric field was $5 \mathrm{~s}$. The lines are to guide the eye.

holds this high voltage suggests impact ionization to be insignificant. Application of the electric field in both directions eventually causes a decrease of the muonium amplitude accompanied by a corresponding increase in the diamagnetic amplitude. From Fig. 2 one can estimate the characteristic electric field (the mid effect field) $E_{c} \sim 8 \mathrm{kV} / \mathrm{cm}$.

In $\mathrm{GaAs},{ }^{17}$ the characteristic electric field was determined to be $E_{c}^{\mathrm{GaAs}} \sim 5 \mathrm{kV} / \mathrm{cm}$. Electrons are very light in GaAs with an effective mass in the $\Gamma$ valley $m^{*} \sim 0.07 m_{e}$. That is why even the ground hydrogenic state in GaAs is rather shallow with energy $U_{1}=-13.6\left(\mathrm{~m}^{*} / \mathrm{m}_{e} \epsilon^{2}\right) \mathrm{eV} \simeq-7 \times 10^{-3} \mathrm{eV}$ (where $\epsilon=11.6$ is the dielectric constant of GaAs). Note that this energy is less than the Debye temperature $T_{D}=344 \mathrm{~K}$ (Ref. 21) in GaAs and an electron can easily loose its energy by a one-phonon emission cascade process starting in the conduction band and going down through the sequence of excited hydrogenic states. By increasing the external electric field one steadily removes excited states with quantum numbers $n$, according to the expression for successive thresholds in electric field

$$
E_{n}=\frac{1}{n^{4}} \frac{\left(m^{*} / m_{e}\right)^{2}}{\epsilon^{3}} \frac{e^{5} m_{e}^{2}}{4 \hbar^{4}}<E .
$$

Equation (3) is directly derived from Eq. (1) by substituting the appropriate expressions for the radius and the energy of the excited state with quantum number $n$ within the hydrogenic model. ${ }^{18}$ At low fields, the electron transitions from the conduction band to the states with small quantum numbers with one-phonon emission are still possible. In GaAs the situation becomes critical when the lowest hydrogenic state with $n=1$ is ionized in electric fields higher than $E_{1}$ $\sim 5 \mathrm{kV} / \mathrm{cm}$. In this case the cross section for the electron capture by the muon is dramatically reduced and muonium formation is prohibited.
The electron effective mass is rather large in CdS $\left[m^{*}\right.$ $=0.2 m_{e}$ (Ref. 22) $]$ while the dielectric constant $[\epsilon=9.1$ (Ref. 23)] is smaller than in GaAs. This should result in a much deeper hydrogenic state in $\mathrm{CdS}\left[U_{1}\right.$ $\left.=-13.6\left(m^{*} / m_{e} \epsilon^{2}\right) \mathrm{eV} \simeq-3.5 \times 10^{-2} \mathrm{eV}\right]$ in comparison with GaAs, with a correspondingly larger electric field required to ionize the lowest hydrogenic state with $n=1\left(E_{1}\right.$ $\sim 100 \mathrm{kV} / \mathrm{cm}$ ). This value is clearly inconsistent with $E_{c}$ $\sim 8 \mathrm{kV} / \mathrm{cm}$ observed experimentally (see Fig. 2). However, electric field required to ionize the first excited hydrogenic state or the state with quantum number 2 in $\mathrm{CdS}$ is $E_{2}$ $=E_{1} / 16 \sim 6 \mathrm{kV} / \mathrm{cm}$ [see Eq. (3)], much closer to the experimental value.

This result can be explained by considering the cascade model for the electron capture by a positive center. ${ }^{14,15}$ The Debye temperature $T_{D}=250-300 \mathrm{~K}$ in CdS (Ref. 24) is smaller than the binding energy of the ground hydrogenic state $-U_{1} \simeq 3.5 \times 10^{-2} \mathrm{eV}=390 \mathrm{~K}$ and direct electron transition from the conduction band to the level with $n=1$ involving one phonon is unlikely. The energy of the level with $n=2$ is $-U_{2}=-U_{1} / 4 \simeq 100 \mathrm{~K}$, which is smaller than the Debye temperature and electron transitions with emission of one phonon from the conduction band and from any level with $n>2$ to the level with $n=2$ are possible. By increasing a still relatively small electric field one steadily removes excited states from the hydrogenic excitation spectrum [see Eq. (3)], however, electrons can still be captured by one-phonon emission to one of the unionized levels. The cross section for the electron capture (or muonium formation) is dramatically reduced when the last state for which one-phonon transitions from the conduction band are possible is removed from the excitation spectrum. In the case of CdS, this "bottleneck" of the capture process is the first excited hydrogenic level (or the level with $n=2$ in our notation).

Note that the final muonium state in CdS (which is detected as a state with an extremely low hyperfine constant) may be deep. In (Ref. 20) this state was attributed to the ground state hydrogenic muon-electron complex (the state with $n=1$ in our notation). The interplay of the electron energy spectrum and the Debye temperature may result in the situation where the electron cannot be captured directly to the lowest hydrogenic state but can still be captured into an excited hydrogenic state. The excited states will then determine the temperature dependence of the muonium fraction: it is more likely that the electron is thermally ionized from one of the excited states than from the deep one. This scenario of muonium ionization is supported by the observation of a remarkable shift in the muon precession frequency in $\mathrm{CdS}$ around the ionization temperature due to atomic diamagnetism. ${ }^{25}$ The interpretation of the value of this shift involves an excited hydrogenic state with macroscopic-sized electronic orbits which scales as $n^{2}$. It should be noted that the characteristic size of an electron orbit extracted from the diamagnetic shift ( $a \sim 100 a_{0}$, where $a_{0}$ is Bohr radius) is also consistent with an $n=2$ excited muonium state.

In conclusion, we have used the technique of $\mu$ SR measurements in alternating electric fields to study the process of electron capture by the positive muon in semi-insulating 
CdS. The results suggest capture of a free electron to excited hydrogenic states by the positive muon. These excited states may determine the subsequent dynamics of the final muonium state in a semiconductor.
This work was supported by NATO (through Grant No. PST.CLG.977687), NSF (Grant No. DMR-0209727), the INTAS Foundation (through Grant No. 97-30063) and the Royal Society of London.
*Present address: Physik-Institut der Universität Zürich, CH-8057 Zürich, Switzerland and Paul Scherrer Institut, CH-5231 Villigen PSI, Switzerland; Electronic address: dimitry.eshchenko@psi.ch

${ }^{1}$ Hydrogen in Semiconductors, edited by N.H. Nickel (Academic Press, New York, 1999).

${ }^{2}$ A. Schenck, Muon Spin Rotation: Principles and Applications in Solid State Physics (Adam Hilger, Bristol, 1986).

${ }^{3}$ B.D. Patterson, Rev. Mod. Phys. 60, 69 (1988).

${ }^{4}$ K.H. Chow, B. Hitti, R.F. Kiefl, in Identification of Defects in Semiconductors, edited by M. Stavola, Vol. 51A of Semiconductors and Semimetals (Academic Press, New York, 1998), pp. 137-207.

${ }^{5}$ D.G. Thomas and J.J. Lander, Chem. Phys. 25, 1136 (1956).

${ }^{6}$ A.R. Hutson, Phys. Rev. 108, 222 (1957).

${ }^{7}$ J.M. Gil, H.V. Alberto, R.C. Vilao, J. Piroto Duarte, N. Ayres de Campos, A. Weidinger, J. Krauser, E.A. Davis, S.P. Cottrell, and S.F.J. Cox, Phys. Rev. B 64, 075205 (2001).

${ }^{8}$ V. Storchak, J.H. Brewer, and G.D. Morris, Phys. Rev. Lett. 75, 2384 (1995).

${ }^{9}$ V. Storchak, J.H. Brewer, and G.D. Morris, Phys. Rev. Lett. 76, 2969 (1996).

${ }^{10}$ D.G. Eshchenko, Ph.D. thesis, Kurchatov Institute, Moscow, 1996.

${ }^{11}$ V.G. Storchak, J.H. Brewer, and D.G. Eshchenko, Appl. Magn. Reson. 13, 15 (1997).

${ }^{12}$ D.G. Eshchenko, V.G. Storchak, J.H. Brewer, G.D. Morris, S.P. Cottrell, and S.F.J. Cox, Phys. Rev. B 66, 035105 (2002).
${ }^{13}$ D.G. Eshchenko, V.G. Storchak, J.H. Brewer, and R.L. Lichti, Phys. Rev. Lett. 89, 226601 (2002).

${ }^{14}$ M. Lax, J. Phys. Chem. Solids 8, 66 (1959).

${ }^{15}$ M. Lax, Phys. Rev. 119, 1502 (1960).

${ }^{16}$ H. Gummel and M. Lax, Ann. Phys. (N.Y.) 2, 28 (1957).

${ }^{17}$ D.G. Eshchenko, V.G. Storchak, and G.D. Morris, Phys. Lett. A 264, 226 (1999).

${ }^{18}$ V.G. Storchak, D.G. Eshchenko, R.L. Lichti, and J.H. Brewer, Phys. Rev. B 67, 121201 (2003).

${ }^{19}$ R.S. Knox, Theory of Excitons (Academic Press, New York, 1963).

${ }^{20}$ J.M. Gil, H.V. Alberto, R.C. Vilao, J.P. Duarte, P.J. Mendes, L.P. Ferreira, N. Ayres de Campos, A. Weidinger, J. Krauser, Ch. Niedermayer, and S.F.J. Cox, Phys. Rev. Lett. 83, 5294 (1999).

${ }^{21}$ N.A. Goryunova, Complex Diamond-like Semiconductors (Sovetskoye Radio, Moscow, 1968) (in Russian).

${ }^{22}$ N.N. Berchenko, V.E. Krevs, V.G. Sredin, Semiconductor Solid Solutions and Their Applications (Voenizdat, Moscow, 1963) (in Russian).

${ }^{23}$ Physics and Chemistry of II-VI compounds, edited by M. Aven and J.S. Prener (North-Holland, Amsterdam, 1967).

${ }^{24}$ N.Kh. Abrikosov, Semiconductors Compounds, Their Manufacturing and Properties (Nauka, Moscow, 1967) (in Russian).

${ }^{25}$ V.G. Storchak, D.G. Eshchenko, S.P. Cottrell, S.F.J. Cox, E. Karlsson, R. Waeppling, and J.M. Gil, Phys. Lett. A 290, 181 (2001). 